\title{
LINEAR DIFFERENCE EQUATIONS WITH PERIODIC COEFFICIENTS
}

ADAIKALAM A. GNANADOSS

Tomlinson Fort [1] ${ }^{1}$ has discussed in detail, by using an ingenious idea, the nature of the solution of this type of equations of the first and second orders; and incidentally has shown that: If

$$
y(i+2)+M(i) y(i+1)+y(i)=0
$$

and

$$
M(i+\omega)=M(i)
$$

then

$$
y(i+\omega)+y(i-\omega)=2 A y(i),
$$

where $A$ is a constant and $i$ and $\omega$ are, of course, integers.

In other words " $A$ second order equation with a periodic coefficient of period $\omega$ can be transformed into an equation of order $2 \omega$ with constant coefficients."

Here the generalization of this result for any order is proved by using an entirely different method of approach.

In what follows $x$ is the independent integral variable and the period $\lambda$ is naturally a positive integer.

Theorem. A linear difference equation of order $n$ with periodic coefficients of common period $\lambda$ can be transformed into a linear difference equation of order $n \lambda$ with constant coefficients.

Or more precisely: If

$$
\begin{aligned}
& \left\{\sum_{i=0}^{n} a_{i}(x) E^{i}\right\} u(x)=0, \\
& a_{i}(x+\lambda)=a_{i}(x) \quad(i=0,1, \cdots, n),
\end{aligned}
$$

then there exist constants $C_{i}(i=0,1, \cdots, n)$ such that

$$
\left\{\sum_{i=0}^{n} C_{i} E^{a}\right\} u(x)=0 .
$$

Proof. Case (1) $\lambda \leqq n$. Without loss of generality we may assume that $a_{n}(x)=-1$. Then using an idea of Milne-Thompson [2] we may

Received by the editors September 25, 1950.

${ }^{1}$ Numbers in brackets refer to the references at the end of the paper. 
write

(3)

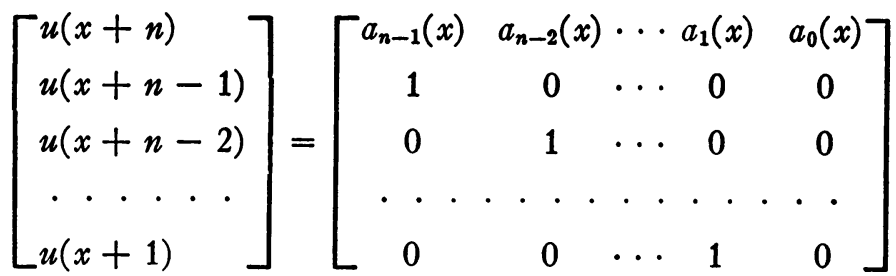

$$
\begin{aligned}
& \times\left[\begin{array}{l}
u(x+n-1) \\
u(x+n-2) \\
u(x+n-3) \\
\cdot \cdot \cdot \cdot \cdot \\
u(x)
\end{array}\right] \\
& =\left[a_{r s}(x)\right]\left[\begin{array}{c}
u(x+n-1) \\
u(x+n-2) \\
\cdot \cdot . \cdot . \\
u(x)
\end{array}\right] .
\end{aligned}
$$

Equation (3) defines $\left[a_{r s}(x)\right]$ and shows that

$$
\left[a_{r s}(x+\lambda)\right]=\left[a_{r s}(x)\right] .
$$

By successive applications of (3) we get

$$
\left[\begin{array}{c}
u(\lambda+x+n-1) \\
u(\lambda+x+n-2) \\
\cdot \cdot \cdot \cdot \cdot \cdot \\
u(\lambda+x)
\end{array}\right]=\left[a_{r s}(x+\lambda-1)\right] \times \cdots
$$

$$
\begin{aligned}
& \times\left[a_{r s}(x+1)\right]\left[a_{r s}(x)\right]\left[\begin{array}{c}
u(x+n-1) \\
u(x+n-2) \\
. . . . \\
u(x)
\end{array}\right] \\
& =\left[b_{r s}(x)\right]\left[\begin{array}{l}
u(x+n-1) \\
u(x+n-2) \\
\cdot . . . \\
u(x)
\end{array}\right] .
\end{aligned}
$$

Equation (4) defines $\left[b_{r s}(x)\right]$ and by (3.1) shows that 


$$
\left[b_{r s}(x+\lambda)\right]=\left[b_{r s}(x)\right] .
$$

By successive applications of (4) and (4.1) we get

$$
\left[\begin{array}{c}
u(i \lambda+x+n-1) \\
u(i \lambda+x+n-2) \\
\cdot \cdot \cdot \cdot \cdot \cdot \\
u(i \lambda+x)
\end{array}\right]=\left[b_{r s}(x)\right]^{i}\left[\begin{array}{c}
u(x+n-1) \\
u(x+n-2) \\
\cdot . \cdot \cdot \cdot \\
u(x)
\end{array}\right] .
$$

Now let the $n$-dimensional vector $v_{i}$ be defined by

$$
\boldsymbol{v}_{i}=\left[\begin{array}{c}
u(i \lambda+x+n-1) \\
u(i \lambda+x+n-2) \\
\cdot \cdot \cdot \cdot \cdot \cdot \\
u(i \lambda+x)
\end{array}\right]
$$

Let $T$ be the homogeneous linear transformation whose matrix is (7)

$$
\left[b_{r s}(x)\right] \text {. }
$$

Then, since the $(n+1)$ th vector $v_{n}$ is a linear function of the $n$ vectors $v_{0}, v_{1}, \cdots, v_{n-1}$ (assumed to be linearly independent), there exist $c_{i}(x)(i=0,1, \cdots, n)$ such that

$$
\sum_{i=0}^{n} c_{i}(x) v_{i}=0
$$

Then from equation (5) it follows that

$$
\left\{\sum_{i=0}^{n} c_{i}(x) T^{i}\right\} v_{0}=0
$$

From equation (9) it is clear that the functions $c_{i}(x)$ are functions of the coefficients of $T^{i}(i=1,2, \cdots, n)$ and not of the components of the vectors. Therefore the functions $c_{i}(x)$ are functions of the elements of $\left[b_{r s}(x)\right]$ alone. Hence by (4.1)

$$
c_{i}(x+\lambda)=c_{i}(x) \quad(i=0,1, \cdots, n) .
$$

Equating the components of (8) separately to zero, we get

$$
\sum_{i=0}^{n} c_{i}(x) u(i \lambda+x+j)=0 \quad(j=0,1, \cdots, n-1) .
$$

Now put $x=k \lambda$, so that $c_{i}(x)=c_{i}(k \lambda)=c_{i}(0)$ by equation (10). The equation (11) becomes 


$$
\sum_{i=0}^{n} c_{i}(0) u(i \lambda+k \lambda+j)=0 \quad(j=0,1, \cdots, n-1) .
$$

But $\lambda \leqq n$ and $j=0,1, \cdots,(n-1)$, and so any $x$ is of the form $k \lambda+j$. Hence the last equation becomes

$$
\sum_{i=0}^{n} c_{i}(0) u(i \lambda+x)=0
$$

which proves the required result (2).

Case (2) $\lambda>n$. The proof is essentially the same, the primary modification being that equation (3) is replaced by

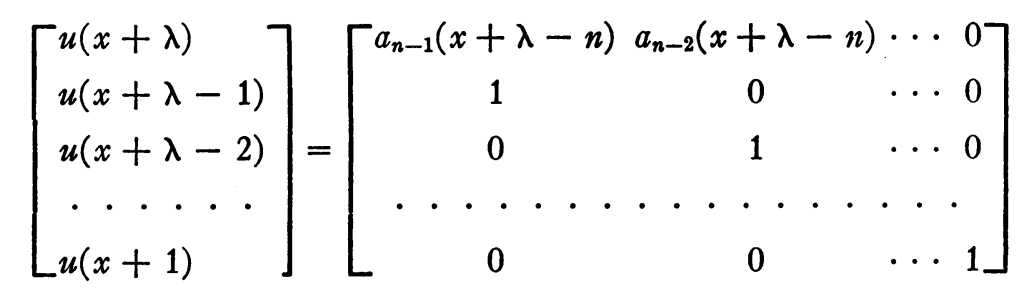

$$
\times\left[\begin{array}{l}
u(x+\lambda-1) \\
u(x+\lambda-2) \\
u(x+\lambda-3) \\
\cdot \cdot \cdot \cdot \\
u(x)
\end{array}\right]
$$

so that the matrix $\left[a_{r s}(x)\right]$ is now $(\lambda-1) \times \lambda$ instead of $n \times n$.

Form of solution. Let

$$
\omega=\cos \frac{2 \pi}{\lambda}+(-1)^{1 / 2} \sin \frac{2 \pi}{\lambda} .
$$

If $\rho^{\lambda}$ is a root, repeated $p$ times, of the equation

$$
\sum_{i=0}^{n} c_{i} z^{i}=0
$$

then the corresponding roots of the auxiliary equation of equation (2) are

$$
\rho, \rho \omega, \rho \omega^{2}, \cdots, \rho \omega^{\lambda-1} \text { each repeated } p \text { times. }
$$

The corresponding terms in the general solution of equation (2) are 


$$
\rho^{x}\left\{\sum_{j=0}^{\lambda-1} \sum_{i=0}^{p-1} A_{i j} x^{i} \omega^{j x}\right\}
$$

Therefore the solution of equation (2) and hence that of (1) will contain terms of the form

$$
\rho^{x} x^{i}\left(A \cos \frac{2 \pi j x}{\lambda}+B \sin \frac{2 \pi j x}{\lambda}\right)
$$

where $\rho, A, B$ are constants and $i, j$ are integers.

But the solution of equation (2) will have $n \lambda$ arbitrary constants; and hence when this solution becomes a solution of equation (1) also there will be $n(\lambda-1)$ equations connecting the $n \lambda$ arbitrary constants.

\section{REFERENCES}

1. Tomlinson Fort, Finite differences, 1948, p. 205.

2. L. M. Milne-Thompson, Finite differences, 1933, p. 379.

The Madras Christian College 